\title{
Structure-Property Relationships of Topological Insulator Nanomaterials
}

Pengzi Liu ${ }^{1,2}$, Judy J. Cha ${ }^{1,2,3 *}$

1. Dept. of Mechanical Engineering and Materials Science, Yale. University, New Haven, USA

2. Energy Sciences Institute, Yale West Campus, West Haven, USA

${ }^{3}$ Canadian Institute for Advanced Research Azrieli Global Scholar, Canada

* Corresponding author: judy.cha@yale.edu

The last decade has witnessed an explosion of a new frontier in condensed matter physics: topological materials with an electronic band structure belonging to a different topological class than ordinary insulators and metals [1]. The non-trivial band topology gives rise to robust, spin-polarized electronic states with linear energy-momentum dispersion at the edge or surface of the materials. For topological materials to be useful in electronic devices, precise control and accurate detection of the topological states are needed in nanostructures, which can enhance the topological states.

The current challenge in the field is to ensure that the crystal quality of topological nanomaterials is sufficiently good in order to easily reveal and control the topological properties. For example, the binary chalcogenide topological insulators such as $\mathrm{BI}_{2} \mathrm{Se}_{3}$ and $\mathrm{Bi}_{2} \mathrm{Te}_{3}$ suffer from chalcogen vacancies and surface oxidation [2], which increase the undesirable bulk carrier densities and decrease the electron mobility, detrimental to the detection of the topological edge states. In addition to the crystal quality, understanding the crystal structure - transport property relationships of topological nanomaterials is critical. For SnTe, a topological crystalline insulator, the required cubic crystal symmetry that provides the topological protection gets lost at low temperatures because SnTe goes through a structural transition from the cubic to the rhombohedral structure [3]. Thus, transmission electron microscopy and spectroscopy is critical for characterization of topological nanomaterials. The structural characterization obtained by electron microscopy must be then correlated to transport measurements to overcome the current challenge.

In this talk, I will discuss my group's efforts in making superconducting SnTe nanowires [4], a candidate material for 1D topological superconductivity. Superconductivity can be induced in SnTe by doping with indium (In) (Figure 1). SnTe nanostructures are fabricated by metal-catalyzed chemical vapor deposition [3-7]. Transport properties of pristine, In-doped, and alloyed SnTe nanostructures are measured and correlated to structural characterization of the nanostructures, obtained by transmission electron microscopy and spectroscopy.

I will highlight the importance of careful structural and morphology characterizations in understanding the transport properties of topological nanostructures. A few examples will be discussed. We observed a structural transition of $\mathrm{SnSe}_{x} \mathrm{Te}_{1-x}$ nanostructures at a critical concentration of Se doping (Figure 2) [6], which was introduced to reduce the carrier density. We also observe surface defects on SnTe nanostructures, arising from dislocations embedded inside the bulk of SnTe nanostructures, which have consequences for the predicted topological properties [7]. 
[1] X.L. Qi and S. C. Zhang, Rev. Mod. Phys. 83 (2011), 1057.

[2] D. Kong et. al., ACS Nano 5 (2011), p.4698-4703.

[3] J. Shen et al., Nano Lett. 14 (2014), p.4183-4188.

[4] P. Kumaravadivel et al., APL Mater. 5 (2017), 076110.

[5] J. Shen et al., Nano Lett. 15 (2015), pp.3827-3832.

[6] J. Shen et al., Adv. Elec. Mater. 2 (2016), 1600144.

[7] P. Liu et al., J. of Phys. Chem. Solids (2017) DOI:10.1016/j.jpcs.2017.12.016.
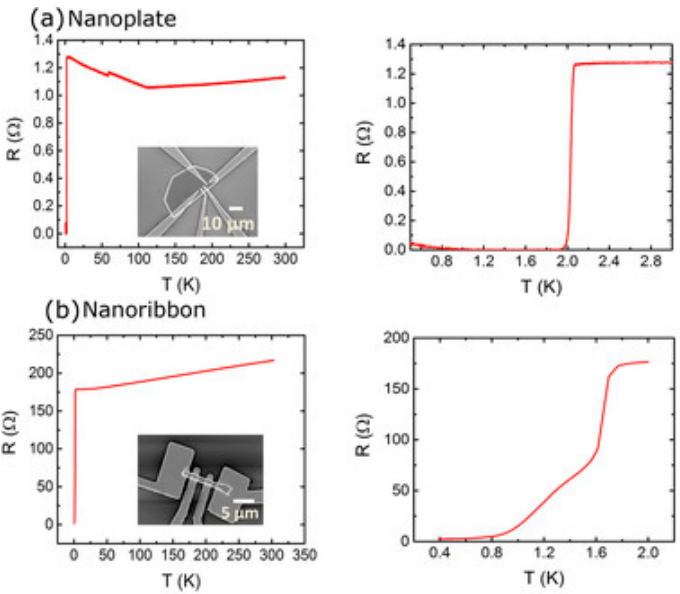

(c) Nanowire
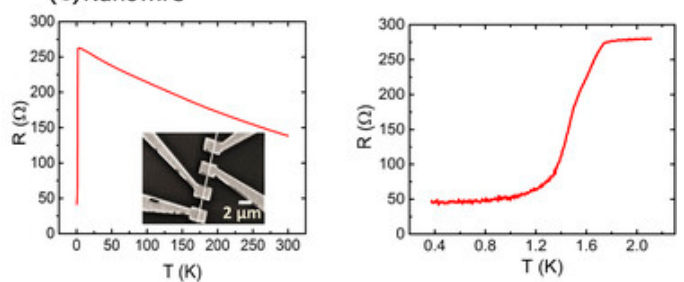

Figure 1. Superconductivity in In-doped SnTe nanostructures: (a) nanoplate, (b) nanoribbon, and (c) nanowire.
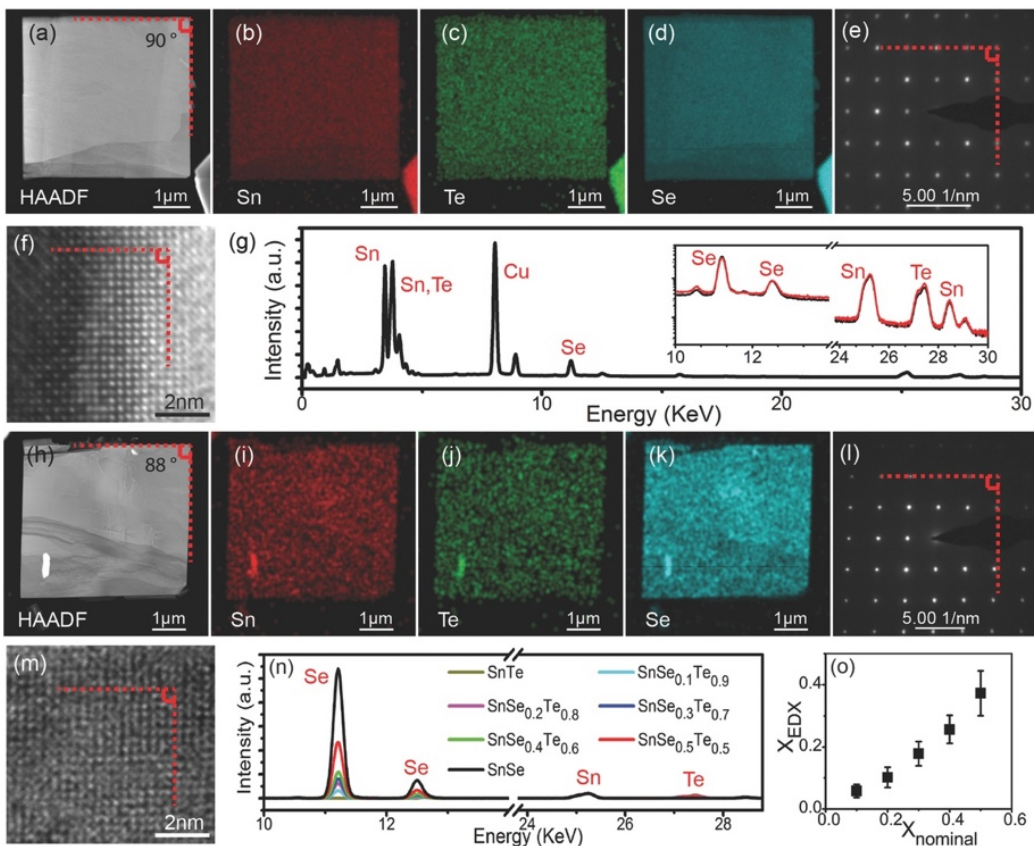

(d)
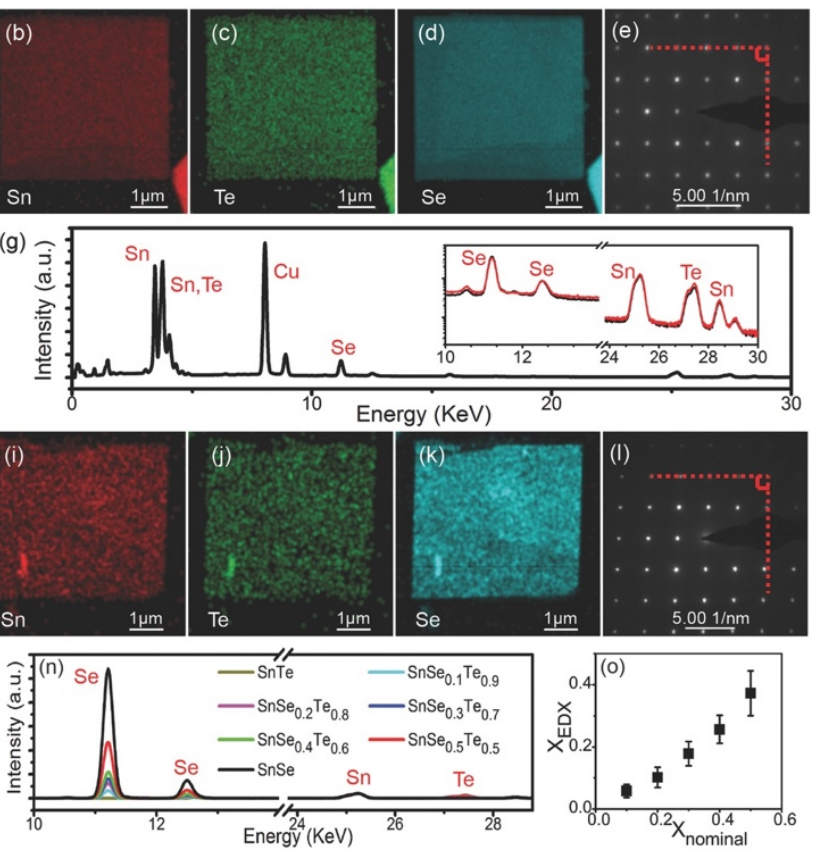

$1 \mu \mathrm{m} \quad \cdot 5.001 / \mathrm{nm}^{\circ}$

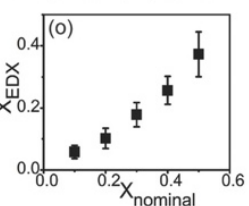

Figure 2. Structural transition observed in $\mathrm{SnSe}_{\mathrm{x}} \mathrm{Te}_{1-\mathrm{x}}$ nanoplates as a function of the Se concentration, $x$. (a-f) pristine SnTe nanoplate with chemical mapping and structural characterization. (g) EDX spectrum to calibrate $\mathrm{x}$. (h-m) $\mathrm{SnSe}_{0.5} \mathrm{Te}_{0.5}$ nanoplate with chemical mapping and structural characterization. (n) EDX spectra of $\mathrm{SnSe}_{\mathrm{x}} \mathrm{Te}_{1-\mathrm{x}}$ nanoplates with varying $x$. (o) Linear correlation between the nominal ratio, $\mathrm{x}$, and the measured ratio, XEDX. 